\title{
The recreational area of the university as a basis for the personal and physical development of students with health related conditions
}

\author{
E. A. Kljushnikova, R. R. Batyrshin, N. A. Batyrshina, and Yu. A. Popkova \\ Tyumen Industrial University, 38 Volodarskogo Street, 625000 Tyumen, Russia
}

\begin{abstract}
In society, both in Russia and abroad, there is a situation with a pronounced tendency towards deterioration in the health of young people, the growth of persons with health related conditions. This problem poses special tasks for the State and society to adapt and to integrate such people into society. The paper examines the possibilities of the recreational area of the university and motor technologies for people with disabilities. The importance of using the recreational environment of the university, built for the needs of both a healthy person and those with disabilities, is emphasized. The research work contains the results of an experiment to improve the musculoskeletal system of students with health related conditions using the conditions of the recreational environment of the university. The practical significance of the work was expressed in the justification of the expediency of using a set of exercises for general physical fitness and special gymnastics for the feet for the physical development of students with health related conditions.
\end{abstract}

\section{Introduction}

In Russia, according to official statistics, there are 12.5 million people with health related conditions; many of them have been diagnosed with disabilities since childhood. Moreover, more than 7 million people with disabilities have access to physical culture and sports [1]. In their works, educators propose to promote discussions on programming and curriculum, to prepare teachers capable of teaching students with disabilities, and to develop special teaching models $[2,3,4]$. The existing system of higher education is little adapted to this category of students, the importance of the recreational environment of the university in the preparation of students with health related conditions (HRC), in which there are potential opportunities for comfortable professional training of such students, is underestimated. S.G. Adyrkhaev notes that "the quality education of young people with different nosologies depends not only on the introduction of modern technologies in the educational process of the university, but also on the environment in which students with health related conditions would feel comfortable" [5]. Such an environment is the university's recreational environment, built to meet the needs of both a healthy person and those with health related 
conditions. There are not many research works devoted to identifying the rehabilitation potential of the educational environment of a university for students with health related conditions. But such issues are beginning to come to the attention of researchers [6]. Physical education in universities as a compulsory academic subject for students with health related conditions exists only formally, but in reality, it is not at all focused on fulfilling a social order-training a young specialist who has a sufficient level of health, the necessary physical education, and physical fitness in order to meet the requirements qualification characteristics of the chosen profession. Not so long ago, the basis of physical education was conditioned by an approach that determined the fulfillment of the average, one-size-fits-all, standards and requirements, which violates the idea of individual human development. In more progressive universities, there has been a change in the paradigm of education aimed at a personality-oriented, active approach, the assimilation of cultural norms and values, which creates adequate conditions for positive shifts in the social and physical spheres of the individual. The scientific novelty of the work lies in the development of a new research hypothesis. Recreational activity in the educational environment of the university is a pedagogical organized motor activity aimed at restoring reduced working capacity using the technologies of health improvement and active recreation, which is especially important for students with HRC [7; 8]. Recreational areas in universities are becoming a reality, contributing to the satisfaction of needs in the process of human life. The quality of education, the state of health, and the general development of the individual depend on the creation of a favorable recreational environment. The healthimproving, psychophysical recreational direction in the educational process of the university is important in maintaining health and provides for the creation of conditions for the restoration of the intellectual and physical strength of students. Achievement of a certain level of physical development of students with HRC by means of physical culture requires the optimal organization of a specialized pedagogical process $[9 ; 10]$.

The purpose of the study was to substantiate the need to use motor recreation technologies for students with HRC in the recreational environment of the university (on the example of the Tyumen Industrial University-TIU). In order to achieve this purpose, it was necessary to solve the following tasks: to describe the technology of motor recreation in the recreational environment of the university; to investigate the functional state of the musculoskeletal system (MSS) in students in the recreational environment of the university; to assess the effectiveness of using a set of exercises of general physical fitness and exercises from the Gymnastics for the Feet Program by T. Lovets for the correction of the state of the musculoskeletal system among students in the recreational environment of the university. The object of the research is the motor recreation of students with HRC. The subject of the research is the technologies of motor recreation of students with HRC.

\section{Materials and methods}

In order to put forward a hypothesis for the study, an analysis of the available biomedical, psychological and pedagogical, methodological literature has been carried out. Methods of mathematical statistics have been used to process the results. For the experiment, a sample of students with HRC was made for research — students with platypodia. Testing of students with HRC has been carried out at an intermediate, control, and ascertaining stage. During the implementation of the complex of exercises of general physical fitness and exercises from the Gymnastics for the Feet Program, the measurement of anthropometric data has been done with the weights, while the handgrip test has been recorded. The study used a mechanical wrist dynamometer DK-50, a mechanical torso dynamometer DS-200, a stopwatch, and a calculator. 


\section{Results and discussions}

The research has been carried out on the basis of the Higher Engineering School (HES) of the TIU group OBB (oil business, bachelor) 1-3 courses. The study has involved students of 1-3 courses of HES, referred for health reasons to a special medical group for physical education. The health limitations of these students are of a functional nature and are defined by a medical diagnosis as the feet functional insufficiency (pes planovalgus).

14 men with platypodia were selected from male students with HRC 18-21 years old. During September 2019 to September 2020, students regularly practiced a specially selected set of exercises for general fitness and exercises from the Gymnastics for the Feet Program. Classes were held 3 times a week in the daytime for an average of 45-60 minutes. From September 2019 to March 2020, they were held in full-time format in the recreational environment of the university, and students were familiarized with the set of exercises and the technique for their implementation. In connection with the transition to distance learning (April-June), classes were conducted through the electronic resource of the university-Educon - with an individual approach to each student and video feedback. Since September, classes have continued in full-time format. During the vacation time (July-August), students completed assignments on their own.

Students were initially tested in September 2019, midterm tests were conducted in March 2020, and benchmarks were conducted in September 2020. We used the methodology of organizing motor recreation classes for students with platypodia in the recreational environment of TIU. Classes were held in the recreational area of TIU, created on the basis of HES, which is a hall with sports equipment and an area for therapeutic exercise and gymnastics. In order to conduct the research, we have compiled and implemented a set of exercises for general physical fitness and used exercises from the Gymnastics for the Feet Program, which is suitable for both children and boys and girls with problems with the foot and ankle. The set of exercises from the Gymnastics for the Feet Program allows to develop the muscles of the foot and ankle, to strengthen the entire lower body, to improve posture, to have a positive effect on the physical development of the body as a whole, and therefore, on the psycho-emotional state. When organizing classes, the rules and safety measures, as well as the established sanitary and hygienic requirements, were strictly observed.

The results were processed mathematically and analyzed, and the main regularities were identified. In order to ensure the motor recreation of students with HRC, a complex of pedagogical conditions has been implemented: the organization of productive pedagogical interaction of physical culture teachers, coaches, curators of student groups, specialists of medical and psychological services of the university; development of methodological support of a motor recreation program for students with HRC; integration of individual characteristics, psychophysical capabilities of interests and needs of students with HRC, creating a comfortable environment, a benevolent atmosphere in the process of educational activity and sectional work on motor recreation; implementation of comprehensive pedagogical, medical, and socio-psychological control; analysis, reflection, and correction of the process of motor recreation of students with HRC.

At the beginning of the study, dynamometry of the right and left hand and torso dynamometry have been carried out.

The obtained results of dynamometry of the right hand have shown that the majority of subjects have an average level- $-72 \%$, a level below average-14\%, a level above average - also $14 \%$ of students.

Dynamometry of the left hand has shown: $72 \%$ - the average level, $1 \%$ - the level below the average, and 3\% - the level above the average.

Dynamometry of the strength of the back muscles has revealed that at the initial stage, 
$14 \%$ of students had a level above average, an average level- $43 \%$, a significant number of subjects - $43 \%$ - had a level below average. Further, the students' flexibility and strength abilities have been tested, according to control tests: the number of push-ups; hitching from suspension on a high bar; raising the torso from a front support position. It was revealed that all students have a level below the average.

In order to increase the level of physical fitness, a complex of gymnastic exercises of general physical fitness and exercises from the Gymnastics for the Feet Program has been implemented.

\section{Research of the level of physical development of students at the intermediate stage}

After six months of systematic training, intermediate testing has been carried out. The dynamometry of the right hand was re-measured. The data obtained at the intermediate stage have shown that the majority of the subjects had an average level- $65 \%$ (instead of $72 \%$ ), but no students with a level below the average were identified, and $35 \%$ had a level above the average instead of $14 \%$ of students.

Dynamometry of the left hand has shown: students with an average level became $65 \%$, the number of students with an indicator above the average have increased from $21 \%$ to $35 \%$. Students with a low level were not identified.

Dynamometry of the strength of the back muscles has revealed that at the intermediate stage, $7 \%$ of students have a higher than average level (instead of $14 \%$ ), but the number of subjects with an average level have increased-from $43 \%$ to $86 \%, 7 \%$ have a level below average - this indicator has decreased from $43 \%$.

At the intermediate stage, the flexibility and physical abilities of students were also tested on control tests: the number of push-ups; hitching from suspension on a high bar; raising the torso from a front support position. Data analysis has shown a positive trend.

Also, the dynamics towards an increase in indicators was revealed in the average indicators of testing physical abilities.

Thus, the positive dynamics revealed by the results of the interim study allows us to conclude about the effectiveness of systematic exercises in general physical fitness and exercises from the Gymnastics for the Feet Program and continue training until the end of the study.

\section{Dynamics of the level of physical development of students with platypodia}

In September 2020, a year after the implementation of a set of exercises of general physical fitness and exercises from the Gymnastics for the Feet Program, a control study of the level of physical development of the same students was carried out.

Table 1. Indicators of physical abilities of students at the control stage

\begin{tabular}{|c|l|c|c|c|c|c|c|c|}
\hline & $\begin{array}{l}\text { Full } \\
\text { name }\end{array}$ & $\begin{array}{c}\text { Dynamometr } \\
\text { y of the right } \\
\text { hand, kg }\end{array}$ & $\begin{array}{c}\text { Dynamomet } \\
\text { ry of the left } \\
\text { hand, kg }\end{array}$ & $\begin{array}{c}\text { Back } \\
\text { streng } \\
\text { th }\end{array}$ & $\begin{array}{c}\text { Flexibilit } \\
\mathbf{y}, \mathbf{c m}\end{array}$ & $\begin{array}{c}\text { Push- } \\
\text { up, kg }\end{array}$ & $\begin{array}{c}\text { Hitchi } \\
\text { ng, kg }\end{array}$ & $\begin{array}{c}\text { Prelu } \\
\text { m, kg }\end{array}$ \\
\hline 1 & $\begin{array}{l}\text { Denis } \\
\text { T. }\end{array}$ & 60 & 55 & 135 & 15 & 43 & 13 & 45 \\
\hline 2 & $\begin{array}{l}\text { Vladi } \\
\text { mir H. }\end{array}$ & 63 & 55 & 140 & 18 & 45 & 13 & 40 \\
\hline 3 & $\begin{array}{l}\text { Alexa } \\
\text { ndr P. }\end{array}$ & 54 & 46 & 135 & 13 & 40 & 6 & 40
\end{tabular}


Table 1. Continued

\begin{tabular}{|c|l|c|c|c|c|c|c|c|}
\hline 4 & $\begin{array}{l}\text { Dmitr } \\
\text { y K. }\end{array}$ & 60 & 55 & 146 & 25 & 30 & 15 & 52 \\
\hline 5 & $\begin{array}{l}\text { Marce } \\
\text { Z. }\end{array}$ & 62 & 55 & 138 & 13 & 15 & 8 & 40 \\
\hline 6 & $\begin{array}{l}\text { Alexa } \\
\text { ndr P. }\end{array}$ & 60 & 55 & 139 & 8 & 20 & 6 & 41 \\
\hline 7 & $\begin{array}{l}\text { Leoni } \\
\text { d L. }\end{array}$ & 50 & 45 & 138 & 15 & 15 & 6 & 38 \\
\hline 8 & $\begin{array}{l}\text { Alexa } \\
\text { ndr N. }\end{array}$ & 47 & 43 & 160 & 16 & 65 & 6 & 60 \\
\hline 9 & $\begin{array}{l}\text { Kirill } \\
\text { O. }\end{array}$ & 53 & 45 & 145 & 8 & 25 & 10 & 51 \\
\hline 10 & $\begin{array}{l}\text { Danila } \\
\text { D. }\end{array}$ & 56 & 50 & 150 & 7 & 27 & 11 & 50 \\
\hline 11 & $\begin{array}{l}\text { Ivan } \\
\text { D. }\end{array}$ & 54 & 45 & 143 & 9 & 45 & 12 & 37 \\
\hline 12 & $\begin{array}{l}\text { Ivan } \\
\text { L. }\end{array}$ & 53 & 46 & 152 & 14 & 65 & 14 & 51 \\
\hline 13 & $\begin{array}{l}\text { Semy } \\
\text { on L. }\end{array}$ & 56 & 50 & 136 & 15 & 40 & 13 & 46 \\
\hline 14 & $\begin{array}{l}\text { Leoni } \\
\text { d L. }\end{array}$ & 56 & $\mathbf{5 6}$ & 150 & 6 & 39 & 10 & 42 \\
\hline $\begin{array}{l}\text { Aggregate } \\
\text { indicator }\end{array}$ & 58 & $\mathbf{1 4 3 . 3}$ & $\mathbf{1 3}$ & $\mathbf{3 6 . 7}$ & $\mathbf{1 0 . 2}$ & $\mathbf{4 5 . 2}$ \\
\hline
\end{tabular}

The functional state of the musculoskeletal system in students with HRC has been studied using tests characterizing the level of development of strength abilities.

In the dynamics of observations among students with platypodia, there is a tendency to an increase in the indicator of the hand dynamometry test (Fig. 1). At the initial stage, the average dynamometry of the right hand in the group of 18-20 year old boys was $43.5 \pm 2.38 \mathrm{~kg}$. Systematic exercises with the selected exercise complexes were characterized by a tendency to increase this indicator at the final stage of the study to $56 \pm 1.12 \mathrm{~kg}$.

The average dynamometry indicator of the left hand in the group of 18-20 years old boys was $40.1 \pm 2.03 \mathrm{~kg}$. There was also a tendency to an increase in this indicator at the final stage of the study to $49.5 \pm 1.12 \mathrm{~kg}$.

At the initial stage, the average indicator of torso dynamometry in the group of young men 18-20 years old was $135.4 \pm 3.8 \mathrm{~kg}$. Systematic exercises with the selected exercise complexes were characterized by a tendency to increase this indicator at the final stage of the study to $143.3 \pm 1.89 \mathrm{~kg}$.

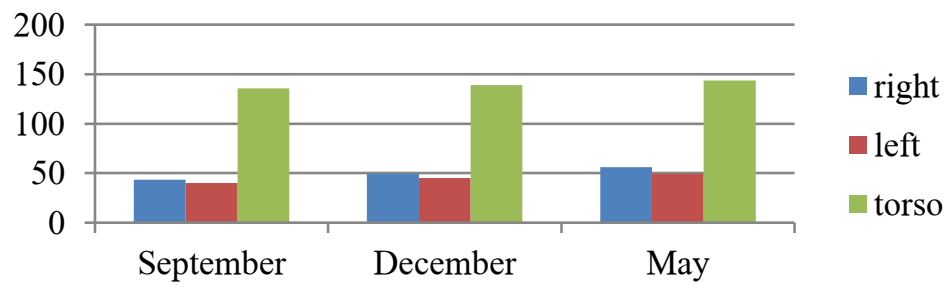

Fig. 1. Dynamometry of the right and left hand, torso

The analysis of individual indicators at the initial stage of the study has revealed that $70 \%$ of students have the average level of strength of the right hand; $15 \%$ of students have the indicator below average, and also $15 \%$ of students have the indicator above average. At the final stage, the number of students with a level above the average has increased to $85 \%$; in this regard, the indicator of the average level was $15 \%$. No students with a level below average were identified. 
Analysis of individual indicators at the initial stage of the study has revealed that $78 \%$ of students have an average level of left hand strength; an indicator is lower than average in $22 \%$ of students. At the final stage, the number of students with an average level of strength has decreased to $65 \%$, but $35 \%$ of students completed exercises above the average level. No students with a level below average were identified.

The data obtained in the course of the study on the average weight of students and dynamometry made it possible to compare the average indicators of the relative strength of the hand. It is expressed as a percentage. To do this, the indicator of the strength of the right hand (as the strongest one) is multiplied by 100 and divided by the indicator of body weight.

Thus, systematic exercises in general physical fitness and exercises from the Gymnastics for the Feet Program by T. Lovets, despite the absence of significant differences in the average group indicator, had a positive effect on the dynamics of the average indicator of relative hand strength among students with HRC from 60, 4\% to $77.7 \%$ (Fig. 2).

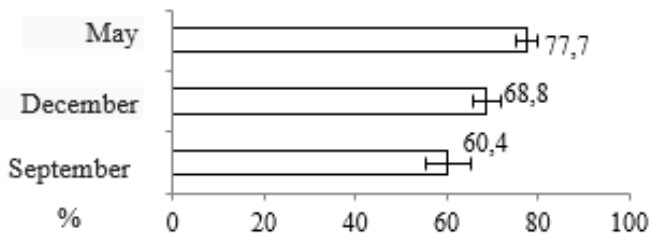

Fig. 2. Dynamics of the average indicator of the relative strength of the hand, \%

Also, the classes have positively influenced the indicators of back muscle strength in students with HRC. The dynamics of the average indicator of the relative strength of the back muscles has ranged from $188 \%$ to $199 \%$ (Fig. 3). The indicator has increased by $5 \%$ within the average.

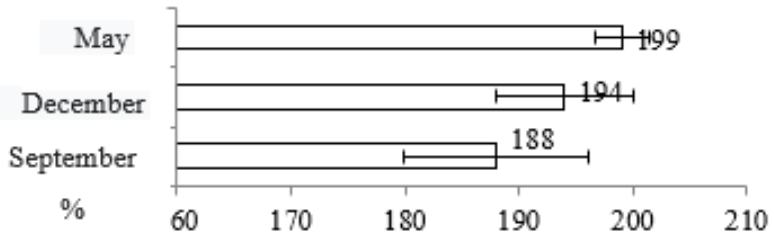

Fig. 3. Dynamics of the average indicator of the relative strength of the back muscles, $\%$

In the dynamics of observations among students with platypodia, there were no significant differences in the indicator of the "Flexibility" test. At the initial stage, the average indicator of flexibility in the group of young men 18-20 years old was $11.2 \pm 1.82 \mathrm{~cm}$. Systematic exercises with the selected exercise complexes were characterized by a tendency to increase this indicator at the final stage of the study to $13 \pm 1.45 \mathrm{~cm}$.

In the dynamics of observations among students with platypodia, there were no significant differences in the indicator of the "Push-ups" test (Fig. 4). At the initial stage, the average indicator of push-ups in the group of 18-20 years old boys was $32.9 \pm 4.28$ times. Systematic exercises with the selected exercise complexes were characterized by a tendency to increase this indicator at the final stage of the study up to $36.7 \pm 4.32$ times. The analysis of individual indicators at the initial stage of the study has revealed that $30 \%$ of students coped with the task as "excellent", $35 \%$ of students completed this test exercise as "good", 35\% received an assessment of "satisfactory", students who did not cope with the control standard, were not found. At the final stage, the number of students who passed the 
test as "excellent" have increased to $50 \%$, mainly due to students who passed the test as "good'- they became 15\%, students who received the mark "satisfactory" have remained $35 \%$, the marks "unsatisfactory" were not found. Thus, systematic exercises in general physical fitness and exercises from the Gymnastics for the Feet Program by T. Lovets, despite the absence of significant differences in the average group indicator, had a positive effect on the individual indicators of the development of strength abilities among students with HRC.

According to the results of observations, no significant difference was also revealed in the indicators of the test "hitching from suspension on a high bar" (Fig. 4). At the initial stage, the average indicator of pull-ups in the group of 18-20 years old boys was $9.2 \pm 0.74$ times. Systematic exercises with the selected exercises were characterized by a tendency to increase this indicator at the final stage of the study up to $10.2 \pm 0.88$ times.

The analysis of individual indicators at the initial stage of the study made it possible to identify $42 \%$ of the students who performed this test exercise as "good", $38 \%$ have received the mark "satisfactory", and $20 \%$ of the students did not cope with the control standard. At the final stage, the number of students who passed the test as "good" has increased to $56 \%$, and those who received the marks "satisfactory" and "unsatisfactory" have decreased to $30 \%$ and $14 \%$, respectively. Thus, systematic exercises in general physical fitness and exercises from the Gymnastics for the Feet Program by T. Lovets, despite the absence of significant differences in the average group indicator, had a positive effect on the individual indicators of the development of upper limb strength in students with HRC.

In the dynamics of observations among students with platypodia, there were no significant differences in the indicator of the test "Raising the torso from a front support position" (Fig. 4).

At the initial stage, the average indicator of raising the torso in the group of 18-20 years old boys was $44.8 \pm 1.84$ times. Systematic exercises with the selected exercises were characterized by a tendency to increase this indicator at the final stage of the study up to $45.2 \pm 1.79$ times.

The analysis of individual indicators at the initial stage of the study allowed to identify $65 \%$ of the students who performed this test exercise as "good", $35 \%$ have received the mark "excellent". At the final stage, the number of students who coped with the standard as "excellent" and "good" remained at the same level. The lack of visible dynamics is explained by the initially rather high level of passing the standards.

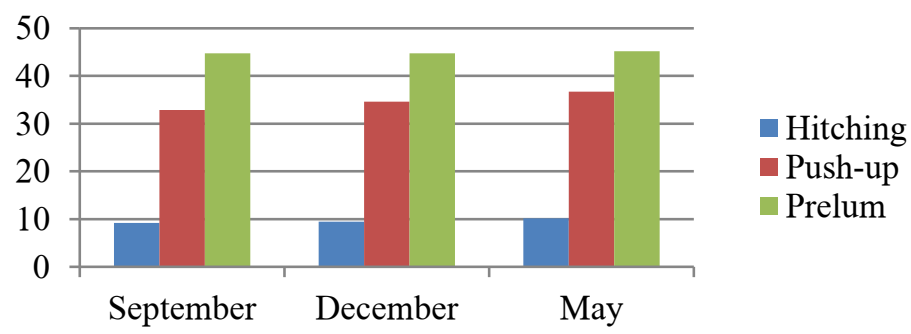

Fig. 4. The influence of physical exercises in the recreational environment of the university $(n=14)$.

\section{Conclusions}

One of the directions of a comfortable educational environment of the university for students with health related conditions is the creation of recreational areas, the main tasks of which are the restoration of physical and moral strength, which forms the basis for personal and physical development. The recreational environment of the university has a 
fairly wide range of effects on the physical and psychosocial sphere of students with health related conditions, and the result in recreational activities is achieved by effective motor exercises, built taking into account health limitations.

In this paper, we have examined the technologies of motor recreation and their use in the educational process of students with health related conditions within the walls of the university. Based on the studied theoretical material, practical material on the research topic has been studied.

The use of traditional means, forms, and methods of physical recreation of students with HRC in the recreational environment of the university does not always ensure the effectiveness of physical development of students with HRC, their recovery, and, as a result, education at different stages of professional training. It was revealed that a differentiated approach to the motor recreation of students is required, in accordance with their health limitations.

In this regard, a set of exercises for general physical fitness and exercises from the Gymnastics for Feet has been used for systematic classes, which were successfully implemented throughout the year in the educational process of students with health related conditions. The results obtained allowed us to make a conclusion about the effectiveness of motor recreation technologies in increasing the level of physical development of students with health related conditions.

The functional state of the musculoskeletal system in students with health related conditions in the dynamics of observation has significantly improved. There was a significant increase in the indicators of the handgrip test (an increase of $18.1 \%, \mathrm{P}<0.05$ ), as well as a tendency to an increase in the indicators of torso dynamometry, in hitching from suspension on a high bar, push-ups in the front support position, flexion of the trunk from the front support position, and mobility in the lumbar spine. The use of a set of exercises of general physical fitness and exercises from the Gymnastics for the Feet Program has turned out to be quite effective for correcting the state of the musculoskeletal system among students with health related conditions in the recreational environment of the university.

Thus, systematic exercises in general physical fitness and exercises from the Gymnastics for the Feet Program, despite the absence of significant differences in the average group indicator, had a positive effect on the individual indicators of strength development in students with health related conditions.

The results obtained indicate a positive trend in all analyzed qualities. The subjects at all stages of the research have qualitatively better results than at the initial stage.

The research confirms the hypothesis of the study that the systematic implementation by students with health related conditions (on the example of students with platypodia) of a set of general physical fitness exercises and exercises from the Gymnastics for the Feet Program in the recreational environment of the university will improve their level of physical development.

\section{References}

1. I. Yamusheva, Chelyabinsk Online (2016) https://74.ru/

2. M.L. Tanure Alves, M. Grenier, J.A. Haegele, E. Duarte, International Journal of Inclusive Education, 24(10), 1129 (2020)

3. Y.A. Hutzler, S.C. Meier, S.D. Reuker, M.A. Zitomer, Physical Education and Sport Pedagogy, 24(3), 249 (2019)

4. O.V. Mamonova, S.I. Filimonova, T.N. Shutova, G.B. Kondrakov, Theory and practice of physical culture, 9, 51 (2018)

5. S.G. Adyrkhaev, Young Researcher, 1(60), 459 (2014) 
6. A.M. Burkova, E.S. Yagudina, B. Ioannis, Theory and practice of physical culture, 5, $50(2020)$

7. L.G. Zaborina, Scholarly Notes of ZabSHPU, 5, 298 (2012)

8. V.P. Zaitsev, Physical Recreation in the Structure of Students' Active Recreation (2013) http://sportfiction.ru/

9. Yu.V. Bessarabova, ASU Bulletin (2014)

10. Yu.V. Bessarabova, ASU Bulletin, 1(193), 103 (2017) 